\title{
Endoscopic Skull Base Suturing with a Self-Closing U-Clip: A Report of Two Cases and Review of the Literature
}

\author{
Peter G. Campbell, MD1, Pranshu Sharma, MD³, Sanjay Yadla, MD', \\ Adam Luginbuhl, MD², Marc R. Rosen, MD², James J. Evans, MD1 \\ 'Department of Neurological Surgery, Thomas Jefferson University, Philadelphia, PA 19107 \\ 2Department of Otolaryngology, Thomas Jefferson University, Philadelphia, PA 19107 \\ ${ }^{3}$ Department of Radiology, Thomas Jefferson University, Philadelphia, PA 19107
}

\begin{abstract}
Objectives: Self-closing nitinol U-clips (Medtronic, Inc., Minneapolis, MN) have primarily been used by vascular surgeons to facilitate vessel anastomosis. The advantage of this device is that it obviates the need for suturing when manipulation of the tissue or needle is prohibitive. The current report describes a novel use of the U-clip as an aid in endoscopic reconstruction of the skull base.

Methods/Participants: Two patients underwent U-clip assisted skull base repair. The first patient underwent transnasal endoscopic repair of an ethmoidal meningoencephalocele requiring primary dural closure and the second underwent odontoidectomy for basilar invagination via an endoscopic transnasal approach requiring primary closure of the soft tissues.

\section{Study Design - Case Series}

Results: The defects were successfully closed using the U-Clip anastomotic device. No cerebrospinal fluid leaks were experienced postoperatively. Intraoperative evaluation combined with postoperative imaging confirmed a satisfactory reconstruction.

Conclusions: The U-clip device may be used as an adjunct in select cases to facilitate endoscopic skull base reconstruction. The use of this tool may prevent tissue migration and enhance dural closure. It may be particularly useful in cases where narrow operative corridors make conventional suturing technically difficult.
\end{abstract}

\section{Abbreviations: CSF-Cerebrospinal Fluid; MRI-Magnetic Resonance Imaging; CT-Computed tomography}

Keywords: cerebrospinal fluid leak, endonasal approach, reconstruction, suture, U-Clip

\section{Introduction}

Endonasal skull base surgical techniques have evolved significantly over the past decade. However, the endoscopic repair of large skull base defects has remained a source of considerable consternation. Widespread acceptance of a purely endoscopic approach to skull base pathology was initially limited by CSF leak rates, which historically ranged from 10 to $58 \%^{1-5}$. More recently however, the reported incidence of high flow intraoperative CSF leaks has varied between $3.3 \%$ and $13.5 \%$, largely due to the advent of pedicled mucosal flaps ${ }^{1,6,7}$. The ultimate goal of reconstructing large skull base defects is not only to prevent the development of CSF fistulas but to create a functional seal between the intracranial and extracranial contents to protect neurologic and ocular function ${ }^{5,8}$.

Unfortunately, the purely endoscopic endonasal approach to the skull base provides a rather limited conduit for the intricate surgical manipulation required for suturing. Several techniques using endoscopic suture as a primary method of securing grafts during skull base reconstruction have been reported but none are widely practiced due their cumbersome nature ${ }^{9,10}$. Recently, newer instruments and techniques have been fashioned for the small, restricted environment frequently encountered during endoscopic approaches ${ }^{5,11-13}$.

The nitinol U-clip (Medtronic, Inc., Minneapolis, MN) was designed to imitate the biomechanical stability of an interrupted suture while eliminating the need for knot tying ${ }^{14}$. By combining its elastic features and thermoshape memory, the nickel titanium alloy U-clip reverts to a predetermined circular shape when exposed to body temperature. This restoration of the closed-loop conformation

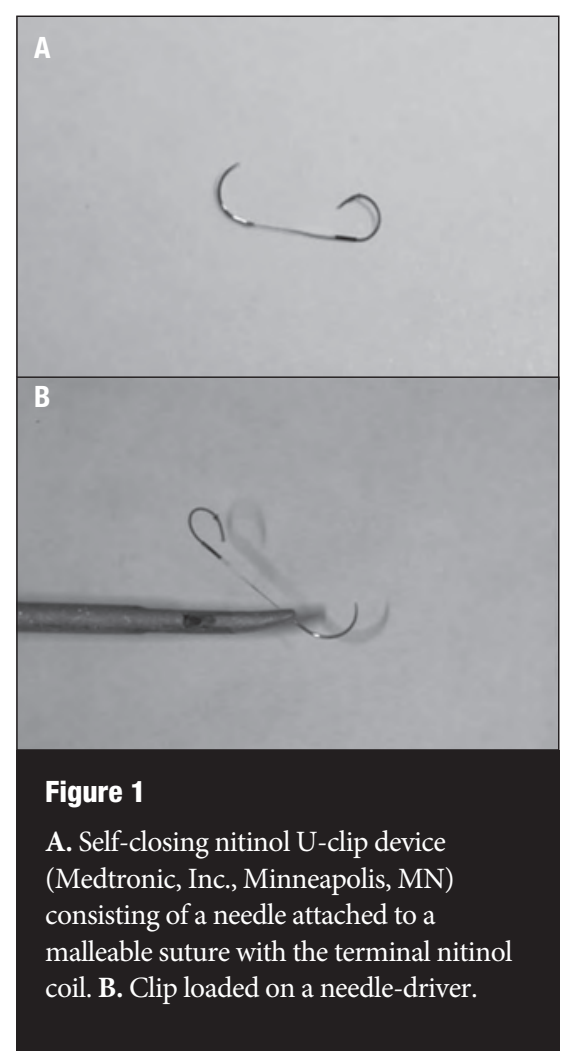

occurs after the device is deployed with the use of a curved needle and a standard needle holder, thus approximating the tissue without any further suturing (Figure 1). The current report describes the use of the U-clip for endoscopic suturing during skull base reconstruction with favorable results.

\section{Methods/Materials}

During skull base reconstruction, the need for intraoperative suturing arose in two patients. The U-clip device was used in these diverse endoscopic cases to aid in the closure of two defects after intervention. In Case 1, the repair was aided by a Durepair (Medtronic, Inc., Minneapolis, MN) onlay. In Case 2, a layer of DuraSeal glue (Confluent Surgical, Inc, Waltham, MA) was applied to the completed closure. One year follow-up was performed for each patient. 

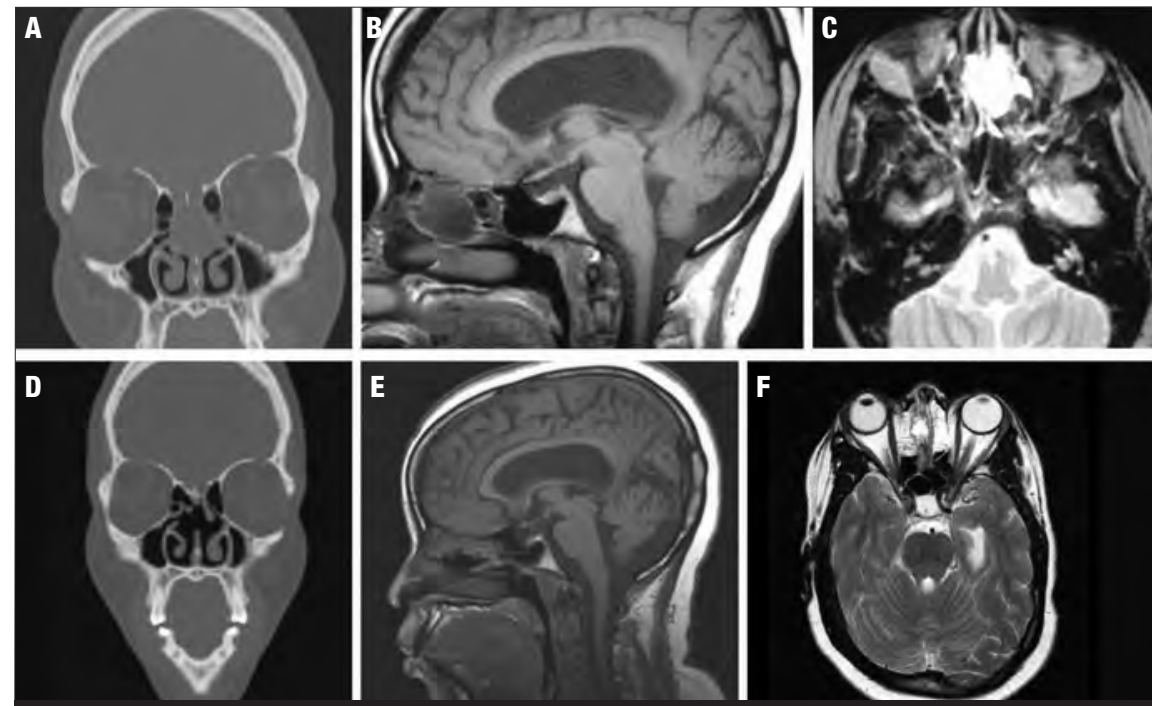

Figure 2

A. Preoperative coronal CT showing anterior skull base defect in a 43-year-old female with a spontaneous transethmoidal meningoencephalocele. B. Preoperative T1-weighted MRI without contrast demonstrates the meningoencephalocele involving the ethmoidal cells and nasal cavity. C. Preoperative axial T2-weighted MRI showing the ethmoidal meningocele. D. Postoperative coronal CT showing the skull base repair. E. T1 MRI without contrast demonstrating separation of the intracranial and extracranial compartments postoperatively F. Postoperative axial T2-weighted MRI showing absence of the ethmoidal meningocele.
A

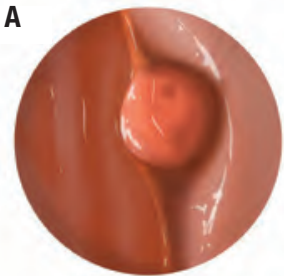

D

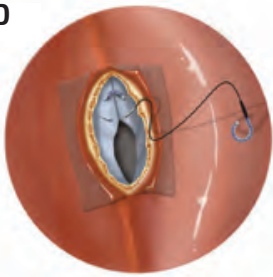

B

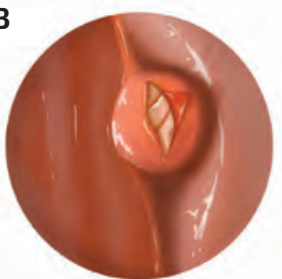

E

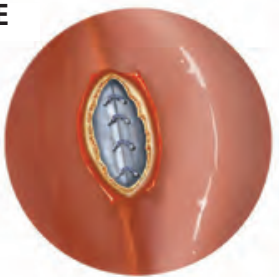

C

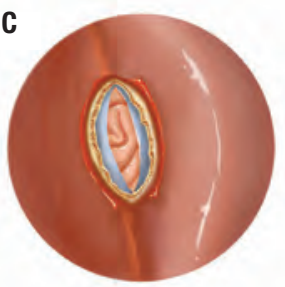

$\mathbf{F}$

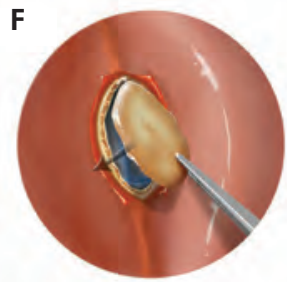

Figure 3

Graphic illustration of the endonasal view of decompression of an ethmoidal meningocele showing A. Meningocele with septum to the right, B. Mucosal opening of the meningocele, C. Dural opening, D. After shrinking the leaflets of the meningocele U-clip is used to re-approximate the dural edges, E. View after dural closure, F. skull base reconstruction with a piece of septal cartiledge.

\section{Results}

Case 1

A 43-year-old female presented with headache, diplopia and spontaneous CSF rhinorrhea. Imaging revealed a $2.1 \times 1.9 \times 1.6 \mathrm{~cm}$ meningoencephalocele in the ethmoid region (Figure 2). The patient underwent an endonasal endoscopic repair. Under endoscopic and navigational visualization, the middle turbinate was resected and the meningocele was identified. After a circumferential dissection of the meningoencephalocele the bony defect was identified in the region of the cribiform plate. The meningocele was then cauterized with bipolar electrocautery and incised, leaving several millimeters of tissue at the pedicle for subsequent repair. Primary closure of the remaining dural leaflets of the meningocele was performed using U-Clip endoscopic sutures (Figure 3). A layer of Durepair was placed in an onlay fashion over the dural repair, and tucked circumferentially underneath the bony defect. A previously harvested fragment of septal cartilage was cut, shaped and placed into the anterior cranial base bony defect and a fibrin sealant was then applied. After the operation, the patient recovered quickly without any signs of CSF rhinorrhea. The postoperative MRI (Magnetic Resonance Imaging) demonstrated a satisfactory anterior cranial base repair with minimal artifact from the U-Clips (Figure 2).

Case 2

A 41-year-old man presented with rapidly progressive symptoms of dizziness, left deltoid and biceps weakness, gait imbalance, and difficulty swallowing. He had undergone an occipito-cervical decompression and instrumented fusion for a Chiari I malformation five years prior. Imaging revealed crowding of the foramen magnum secondary to basilar invagination with interval development of edema within the cervical spinal cord extending from the craniocervical junction to the $\mathrm{C} 6$ vertebral level (Figure 4). The patient underwent an endoscopic transnasal odontoidectomy without durotomy to decompress the underlying neural elements. To reconstruct the defect, the longus coli muscles and soft tissue as well as the mucosa of the nasopharynx were reapproximated in the midline utilizing the U-clip endoscopic suture followed by a small amount of DuraSeal glue applied to the mucosal surface. Post-operative imaging revealed resolution of cord edema and well-healed nasopharyngeal mucosa was seen 

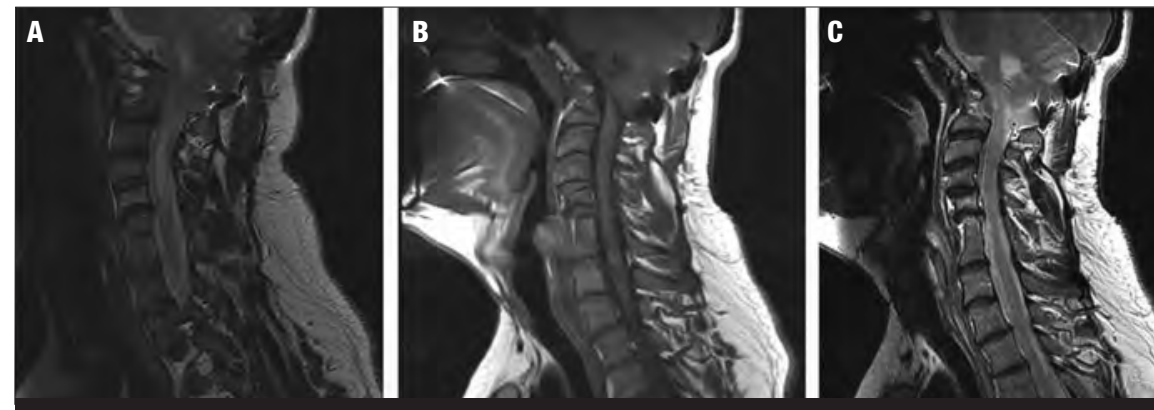

Figure 4

A. Preoperative T2 MRI demonstrating basilar invagination and cord signal change down to the C6 vertebral body. B. Postoperative T1 MRI after transnasal decompression C. T2 MRI showing resolution of cord signal abnormality at 2 weeks.

on endoscopy at follow-up (Figure 4). The patient experienced a full recovery without any complications.

\section{Discussion}

\section{Radiographic Appearance}

Given the neoplastic nature of many skull base lesions and their coincident need for follow-up imaging, any material incorporated into a skull base reconstruction would ideally have minimal associated artifact on CT and retain MRI compatibility. The use of the U-clip device has not affected post-operative imaging in the authors' experience. The device has been rated by the American Society for Testing and Materials as MRI conditional, in that it is an item that has been demonstrated to pose no known hazards in a MRI environment of 3 Tesla or less. However, this device was given a conditional 6 classification, meaning these criteria were determined in non-clinical testing ${ }^{15}$. The U-clip can be seen on bone windows of thin section CT imaging as a tiny curvilinear or circular high density structure. It is not easily confused with bony fragments given its peculiar shape (Figure 5). On MRI, the clip can be difficult to visualize due to its small size and lack of signal. The two patients in the current series underwent frequent post-operative CT and MRI scans without complication.

\section{Endonasal Suturing}

The endonasal approach is performed through a narrow nasal corridor which restricts surgical manipulation. Suturing via this approach can be especially difficult and has been previously characterized as technically demanding ${ }^{16,17}$. However, several authors have described effective transnasal suturing. Kitano and Taneda reported use of a double layered fascial graft sutured to the dural defect to repair skull base defects after tumor resection'. Cukurova et al. reported the successful repair of an ethmoid roof CSF fistula by direct endoscopic suturing and Ahn et al. described an endonasal dural suturing technique using special suture-tying microinstruments ${ }^{10,13}$. Despite these reports, proficiency with these techniques demands an extensive learning process with some authors describing an initial closure time of up to 9 hours ${ }^{13}$.

Secondary to its improved illumination and wide field of view, the endonasal endoscopic approach to the skull base has recently noted an expansion in use and popularity ${ }^{18-20}$. Endoscopic knot tying, similar to endoscopic suturing, has been described as inefficient and impractical ${ }^{5}$. The use of the U-Clip device, initially developed for cardiovascular surgical applications, allows for suturing vessels and viscera, thus eliminating the need for delicate suture manipulation ${ }^{21,22}$. Gardner, et al. previously reported a U-clip based endoscopic dural repair after a skull base meningioma resection $^{5}$. In the authors' experience, the U-clip eliminates the need for endoscopic knot tying, while providing the structural integrity of an interrupted suture.

Other minimally invasive uses for the U-clip

This anastomotic device has found uses in other areas of neurosurgery as well as other specialties $^{14}$. In minimally invasive spine surgery, U-clips have reportedly been used to successfully repair an incidental durotomy after a minimally invasive discectomy ${ }^{23}$. Ferroli, et al. has reported their use in intracranial microanastomoses for high-flow arterial bypasses ${ }^{14,24,25}$. In other surgical arenas, U-clips have been used to enhance suturing in minimally invasive procedures such as laparoscopic gastric banding, laparoscopic repair of perforated peptic ulcers, and robotically assisted mitral valve repair ${ }^{26,27}$.

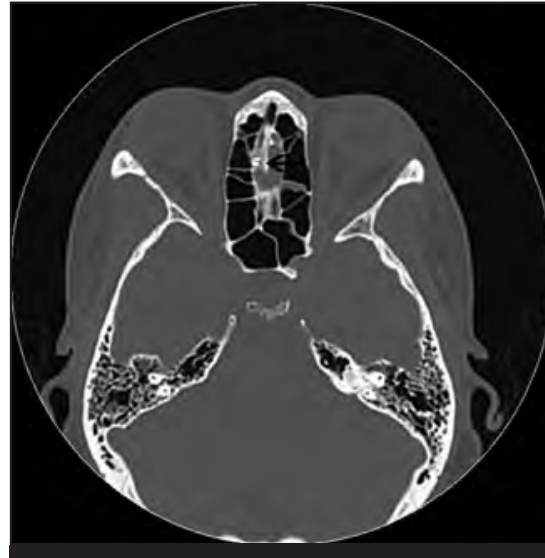

Figure 5

Axial CT of the Head shows the U-clip

(arrow) as a small, curvilinear, high density structure in the roof of right nasal cavity with minimal streak artifact.

The present series describes the use of U-clips for varied endoscopic skull base applications: dural reconstruction and mucosal reapproximation after an endoscopic odontoidectomy. In endoscopic operative fields, the U-clip's ease of use combined with a glacis learning curve establishes it as a functionally diverse device with the ability to safely couple various tissue types while maintaining the stability of an interrupted suture ${ }^{28}$.

\section{Conclusion}

This is the first report describing the use of selfclosing U-clips for closure and reconstruction of the pharyngeal mucosa and muscular layer after an endoscopic odontoidectomy. The U-clip anastomotic device offers an easily-applicable alternative to endoscopic endonasal suturing. While more studies are needed to verify these early results, this penetrating, sutureless anastomotic device seems well-suited for minimally invasive neurosurgical applications when traditional suturing techniques are problematic.

\section{References}

1. de Divitiis E, Cavallo LM, Cappabianca P, Esposito F. Extended endoscopic endonasal transsphenoidal approach for the removal of suprasellar tumors: Part 2. Neurosurgery 2007;60:46-58; discussion 58-49.

2. Dusick JR, Esposito F, Kelly DFet al. The extended direct endonasal transsphenoidal approach for nonadenomatous suprasellar tumors.[see comment]. Journal of Neurosurgery 2005;102:832-841.

3. Frank G, Pasquini E, Doglietto Fet al. The endoscopic extended transsphenoidal approach for craniopharyngiomas. Neurosurgery 2006;59:ONS 75-83; discussion ONS 75-83.

4. Laufer I, Anand VK, Schwartz TH. Endoscopic, endonasal extended transsphenoidal, transplanum transtuberculum approach for resection of suprasellar lesions. Journal of Neurosurgery 2007;106:400-406. 
5. Gardner PA, Kassam AB, Snyderman CHet al. Outcomes following endoscopic, expanded endonasal resection of suprasellar craniopharyngiomas: a case series.[see comment] Journal of Neurosurgery 2008;109:6-16.

6. Zanation AM, Carrau RL, Snyderman CHet al. Nasoseptal flap reconstruction of high flow intraoperative cerebral spinal fluid leaks during endoscopic skull base surgery. Am J Rhinol Allergy 2009;23:518-521.

7. Harvey RJ, Nogueira JF, Schlosser RJ, Patel SJ, Vellutini E, Stamm AC. Closure of large skull base defects after endoscopic transnasal craniotomy. Clinical article. Journal of Neurosurgery 2009;111:371-379.

8. Liu JK, Niazi Z, Couldwell WT. Reconstruction of the skull base after tumor resection: an overview of methods. Neurosurgical Focus 2002;12:e9.

9. Kitano $M$, Taneda $M$. Subdural patch graft technique for watertight closure of large dural defects in extended transsphenoidal surgery. Neurosurgery 2004;54:653-660; discussion 660-651.

10. Cukurova I, Cetinkaya EA, Aslan IB, Ozkul D. Endonasal endoscopic repair of ethmoid roof cerebrospinal fluid fistula by suturing the dura. Acta Neurochir (Wein) 2008;150:897900; discussion 900 .

11. Kubo S, Hasegawa H, Inui T, Tominaga S, Yoshimine T. Suture knot on the repair splint: a simple method to facilitate reconstruction of the sella turcica during endonasal endoscopic transsphenoidal surgery. Technical note. Journal of Neurosurg 2005;102:938-939.

12. Kawamata T, Amano K, Hori T. Novel flexible forceps for endoscopic transsphenoidal resection of pituitary tumors: technical report. Neurosurg Rev 2008;31:65-68; discussion 68 .

13. Ahn JY, Kim SH. A New Technique for Dural Suturing With Fascia Graft for Cerebrospinal Fluid Leakage in Transsphenoidal Surgery Neurosurgery 2009;65: 65-72.

14. Ferroli P, Biglioli F, Ciceri E, Addis A, Broggi G. Self-closing U-clips for intracranial microanastomoses in high-flow arterial bypass: technical case report. Neurosurgery 2007;60:170
15. Frank G. Shellock PD. www.MRIsafety.com MRI Safety, bioeffects and patient management. Los Angeles, CA, 2009.

16. Freidberg SR, Hybels RL, Bohigian RK. Closure of cerebrospinal fluid leakage after transsphenoidal surgery: technical note. Neurosurgery 1994;35:159-160.

17. Vanaclocha V, Saiz N, Panta F. Repair of dural defects in awkward areas-technical note. Acta Neurochir (Wein) 1998;140:615-618.

18. Dehdashti AR, Ganna A, Karabatsou K, Gentili F. Pure endoscopic endonasal approach for pituitary adenomas: early surgical results in 200 patients and comparison with previous microsurgical series. Neurosurgery 2008;62:1006-1015; discussion 1015-1007.

19. Jho HD, Carrau RL. Endoscopic endonasal transsphenoidal surgery: experience with 50 patients. J Neurosurg 1997;87:44-51.

20. Dehdashti AR, Ganna A, Witterick I, Gentili F. Expanded endoscopic endonasal approach for anterior cranial base and suprasellar lesions: indications and limitations. Neurosurger 2009;64:677-687; discussion 687-679.

21. Ross JR. Creation of native arteriovenous fistulas with interrupted anastomoses using a self-closing clip device - one clinic's experience. J Vasc Access 2002;3:140-146.

22. Guglielminotti P, Bini R, Fontana D, Leli R. Laparoscopic repair for perforated peptic ulcers with U-CLIP(R). World J Emerg Surg 2009;4:28.

23. Ferroli P, Franzini A, Messina G, Tringali G, Broggi G. Use of self-closing U-clips for dural repair in mini-invasive surgery for herniated disc. Acta Neurochir (Wein) 2008;150:1103-1105.

24. Ferroli P, Acerbi F, Tringali G, Polvani G, Parati E, Broggi G. Self-closing Nitinol U-Clips for intracranial arterial microanastomosis: a preliminary experience on seven cases. Acta Neurochir (Wein) 2009;151:969-976; discussion 976.

25. Ferroli P, Ciceri E, Addis A, Broggi G. Self-closing surgical clips for use in pericallosal artery-pericallosal artery side-toside bypass. Journal of Neurosurgery 2008;109:330-334
26. Cook RC, Nifong LW, Enterkin JEet al. Significant reduction in annuloplasty operative time with the use of nitinol clips in robotically assisted mitral valve repair. J Thoracic Cardiovasc Surg 2007;133:1264-1267.

27. Lirici MM, Salerno F, Califano A. The use of superelastic suture clips in laparoscopic gastric banding. Minim Invasive Ther Allied Technol 2008;17:176-180.

28. Demaria RG, Fortier S, Malo O, Carrier M, Perrault LP. Interrupted coalescent nitinol clip versus continuous suture coronary anastomosis: a comparative endothelial function study. Heart Surg Forum 2003;6:72-76.

\section{Corresponding Author:}

Peter G. Campbell, MD

Department of Neurological Surgery

Thomas Jefferson University

909 Walnut St, 3rd Floor

Philadelphia, PA 19107

Phone: 215-503-7008

Email: peter.campbell@jeffersonhospital.org

Level of Evidence: 4 (Case Series)

Financial Disclosure: No author has received any financial support from any company associated with products discussed in this article.

Conflict of Interest: None 